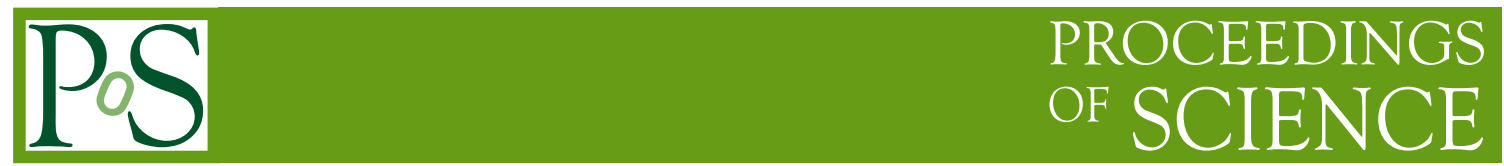

\title{
Holographic reconstruction of scalar field models of dark energy in the background of Brans Dicke cosmology
}

\section{Surajit Chattopadhyay*}

MCA Division, Pailan College of Management and Technology, Kolkata, India

E-mail: surajitchatto@outlook.com

\begin{abstract}
In this work we considered the generalized Brans-Dicke (BD) model in which the scalar field couples non-minimally with matter sector. Extended holographic Ricci dark energy (EHRDE) has been considered in the above framework of BD cosmology. A stronger matter-chameleon coupling has been observed with expansion of the universe. Considering a correspondence between the reconstructed holographic dark energy and scalar field models of dark energy we have reconstructed the corresponding equation of state parameters and observed that it is possible to attain the "phantom" phase of the universe under this reconstruction.
\end{abstract}

The European Physical Society Conference on High Energy Physics

22-29 July 2015

Vienna, Austria

*Speaker.

${ }^{\dagger}$ Financial support from DST, Govt of India under grant no. SR/FTP/PS-167/2011 is thankfully acknowledged. 


\section{Introduction}

Accelerated expansion of the current universe has been observationally established by $[1,2]$. This accelerated expansion of the universe is usually attributed to the presence of an exotic kind of energy, called "dark energy" (DE). The physical origin of the DE is one of the largest mysteries not only in cosmology but also in fundamental physics [3, 4, 5, 6, 7]. DE is characterized by negative pressure and the equation of state (EoS) parameter $w_{D E}=p_{D E} / \rho_{D E}$ has to be less than $-1 / 3$ for accelerated expansion. If $w_{D E}<-1$ then the model is categorized as "phantom", if $w_{D E}>-1$ then the model is characterized as "quintessence". If there is a transition from quintessence to phantom, then is model is categorized as "quintom". The simplest candidate for DE is the cosmological constant $\Lambda$ for which the EoS parameter $w_{\Lambda}=-1$. Although $w_{\Lambda}=-1$ is supported by observations, $\Lambda$ can not explain the time time evolution of the EoS parameter. Because of this limitation various dynamic models of DE have been proposed and such models have EoS parameter evolving with cosmic time $t$. Such models of DE can be categorized as (i) Chaplygin gas models, (ii) scalar field models and (iii) holographic DE models.

Another approach to explain this acceleration is the "modified gravity approach", where the left hand side of Einstein's equations is modified. This approach has been reviewed in $[8,9]$. The evidence of these theories is related to extension of the Einstein-Hilbert action. This occurred by introducing some curvature invariants of higher order and/or minimally or non-minimally coupled scalar fields to the dynamics. The simplest modification of this action is $f(R)$ theory which mainly explore the acceleration of the expanding universe and provide modification of the Einstein theory on large scales.

In recent times, scalar tensor theories have been considered extensively and one important example of the scalar tensor theories is the Brans-Dicke (BD) theory of gravity which was introduced by Brans and Dicke [10] to incorporate the Mach's principle in the Einstein's theory of gravity. Popularity behind BD modified gravity $[11,12]$ lies in the fact that it naturally arises as the low energy limit of many other quantum gravity theories, like the Kaluza-Klein one or the superstring theory.

In the present work we are considering a special form of HDE [13] dubbed as "extended holographic Ricci dark energy" (EHRDE) [14], whose density has the form

$$
\rho_{\Lambda}=3 M_{p}^{2}\left(\alpha H^{2}+\beta \dot{H}\right)
$$

where, $M_{p}^{2}$ is the reduced Planck mass, $\alpha$ and $\beta$ are constants to be determined. Like ref. [15], we shall assume that $\rho_{\Lambda}$ and pressureless dark matter are conserved separately and we shall assume a non-minimal coupling between the scalar field and the matter field.

\section{EHRDE in chameleon BD cosmology}

We consider chameleon BD theory in which the scalar field is coupled non-minimally to the matter field via the action [16]

$$
S=\frac{1}{2} \int d^{4} x \sqrt{-g}\left(\phi R-\frac{\omega}{\phi} g^{\mu \nu} \nabla_{\mu} \phi \nabla_{\nu} \phi-2 V+2 f(\phi) L_{m}\right)
$$


where $R$ and $\phi$ denote the Ricci scalar and BD scalar field respectively. The $f(\phi)$ and $V(\phi)$ are analytic functions of the scalar field. The matter Lagrangian density, denoted by $L_{m}$, is coupled with $\phi$ via the function $f(\phi)$. In a spatially flat FRW universe the corresponding modified field equations are [16]

$$
\begin{gathered}
3 H^{2}=\frac{f}{\phi} \rho+\frac{\omega}{2} \frac{\dot{\phi}^{2}}{\phi^{2}}-3 H \frac{\dot{\phi}}{\phi}+\frac{V}{\phi} \\
3\left(\dot{H}+H^{2}\right)=-\frac{3 \rho}{\phi(2 \omega+3)}\left[\gamma \phi f^{\prime}+\left(\omega\left(\gamma+\frac{1}{3}\right)+1\right) f\right]-\omega \frac{\dot{\phi}^{2}}{\phi^{2}}+3 H \frac{\dot{\phi}}{\phi}+\frac{1}{2 \omega+3}\left[3 V^{\prime}+(2 \omega-3) \frac{V}{\phi}\right] \\
(2 \omega+3)(\ddot{\phi}+3 H \dot{\phi})-2\left(2 V-\phi V^{\prime}\right)=\rho\left((1-3 \gamma) f+2 \gamma \phi f^{\prime}\right)
\end{gathered}
$$

In equations (2.2) to (2.4), $\rho=\rho_{m}+\rho_{\Lambda}$ and $\gamma=\frac{p_{\Lambda}}{\rho_{m}+\rho_{\Lambda}}$ (since dark matter is pressureless, $p_{m}=0$ ).

We now assume the scale factor as $a(t)=a_{0} t^{n}$; and $\phi(t)=\phi_{0} t^{m}$. We shall use these ansatz to reconstruct the potential $V$ and the analytic function $f$ of the chameleon BD cosmology. On putting these ansatz in equation (2.2) we get an equations that involves $V, f$ and remaining terms as functions of $t$

$$
V=-\frac{1}{a_{0}^{3}} \rho_{m 0} t^{-3 n} f+\frac{\phi_{0} t^{m-2}}{2}\left[-m^{2} \omega+6 n(m+n-(n-\beta) f)\right]
$$

We again differentiate it with respect to $t$ and get a new equation involving $\dot{V}$ and $\dot{f}$. Using this in (2.3) along with the said ansatz we get the following differential equation on $f$ with $t$ as the independent variable.

$$
\frac{d f(t)}{d t}-\frac{3(m+2 n)}{2 t} f(t)=\frac{3 a_{0}^{3} \phi_{0} t^{m+3 n-1}\left(m n(t(3+2 \omega)-4-2 n)+m^{3}(1+\omega)-m^{2}(1+2 n)-4 n^{2}\right)}{2\left(\rho_{m 0} t^{2}-a_{0}^{3}(m-2) \phi_{0}(n \alpha-\beta)\right)}
$$

Solving (2.6) we get reconstructed analytic function $f(\phi)$ as

$$
\begin{gathered}
f(\phi)=f\left(\phi_{0} t^{m}\right)=t^{m+3 n}\left[C_{1} t^{\frac{m}{2}}-\frac{2}{\rho_{m 0}\left(8+6 m+m^{2}\right) t^{2}}\right. \\
\left\{m n(4+m)(3+2 \omega) t \text { Hypergeometric } 2 F 1\left[\frac{2+m}{4-2 m-6 n}, 1, \frac{6(n-1)+m}{2(3 n+m-2)}, \frac{a_{0}^{3}(m-2) \phi_{0}(n \alpha-\beta)}{\rho_{m 0}}\right]\right. \\
+(2+m)\left(-4 n^{2}-2 m n(2+n)-m^{2}(1+2 n)+m^{3}(1+\omega)\right) \\
\left.\left.\times \text { Hypergeometric } 2 F 1\left[\frac{4+m}{4-2 m-6 n}, 1, \frac{6 n-8+m}{2(3 n+m-2)}, \frac{a_{0}^{3}(m-2) \phi_{0}(n \alpha-\beta)}{\rho_{m 0}}\right]\right\}\right]
\end{gathered}
$$

Using (2.7) in (2.5) we get the reconstructed potential function as

$$
\begin{gathered}
V(\phi)=V\left(\phi_{0} t^{m}\right)=\frac{t^{-4+m}}{2 a_{0}^{3}(2+m)(4+m) \rho_{m 0}} \times \\
\left(-(2+m)(4+m) \rho_{m 0} t^{2}\left(2 C_{1} \rho_{m 0} t^{\frac{4+m}{2}}+a_{0}^{3} \phi_{0}\left(-6 m n+6 n\left(-n+C 1 t^{\frac{3}{2}(m+2 n)}(n \alpha-\beta)\right)+m^{2} \omega\right)\right)\right) \\
+4\left(\rho_{m 0} t^{2}+3 a_{0}^{3} n \phi_{0} t^{m+3 n}(n \alpha-\beta)\right) \times \\
{\left[m n(4+m)(3+2 \omega) t \text { Hypergeometric } 2 F 1\left[1, \frac{2+m}{4-2 m-6 n}, 1+\frac{2+m}{4-2 m-6 n}, \frac{a_{0}^{3}(m-2) \phi_{0}(n \alpha-\beta) t^{-2+m+3 n}}{\rho_{m 0}}\right]+\right.} \\
(2+m)\left(-4 n^{2}-2 m n(2+n)-m^{2}(2 n+1)+m^{2}(1+\omega)\right) \\
\left.\times \text { Hypergeometric } 2 F 1\left[1, \frac{4+m}{4-2 m-6 n}, 1+\frac{4+m}{4-2 m-6 n}, \frac{a_{0}^{3}(m-2) \phi_{0}(n \alpha-\beta) t^{-2+m+3 n}}{\rho_{m 0}}\right]\right]
\end{gathered}
$$




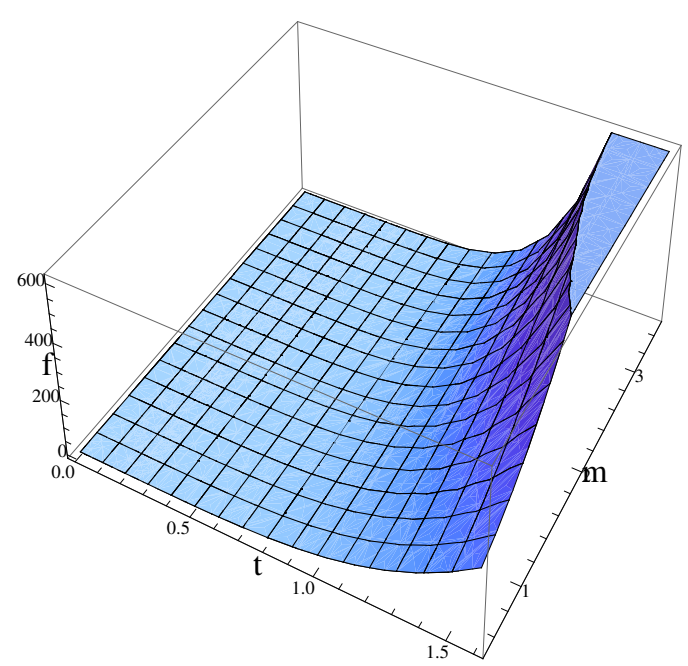

Figure 1: Reconstructed $f(\phi)$ against $t$ for a range of values of $m$.

In Fig. 1 we have plotted EHRDE reconstructed coupling function $f(\phi)$ against $t$ for a range of positive values of $m$. The increasing pattern of $f(\phi)$ with cosmic time $t$ indicates that with the expansion of the universe, the matter-chameleon coupling gets stronger.

\section{Reconstruction of DBI-essence dark energy model}

During the last few years, new ideas in string theory based on the concept of branes have revealed themselves especially fruitful. In particular, scenarios where the inflation is interpreted as the distance between two branes moving in the extra dimensions along a warped throat have given rise to many interesting studies [17]. Martin and Yamaguchi [17] investigated whether the same kind of ideas can lead to sensible dark energy scenarios and introduced a scalar-field model of dark energy having a scalar field with a non-standard Dirac-Born-Infeld (DBI) kinetic term.

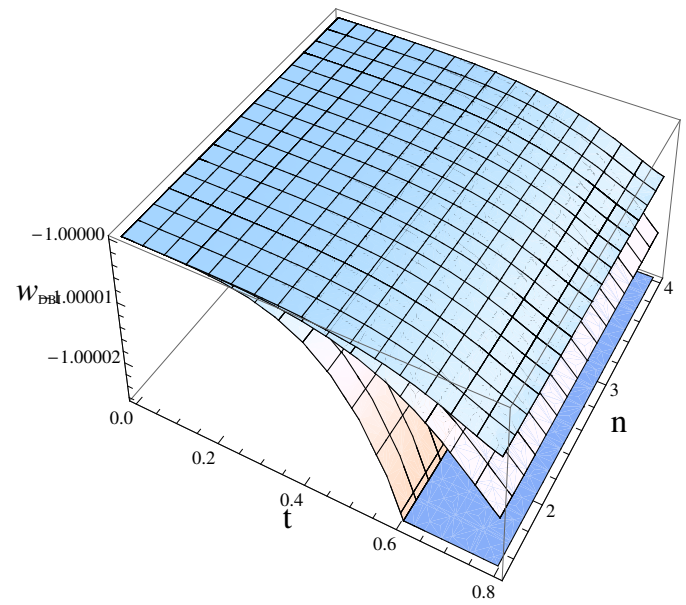

Figure 2: Reconstructed $w_{D B I}$ against $t$ for a range of values of $n$. 
This model is dubbed as "DBI-essence dark energy" and the energy density and the pressure of the DBI-essence model are given, respectively, by [17]:

$$
\begin{aligned}
& \rho_{D B I}=(\eta-1) T(\varphi)+V(\varphi), \\
& p_{D B I}=\left(\frac{\eta-1}{\eta}\right) T(\varphi)-V(\varphi),
\end{aligned}
$$

where:

$$
\eta=\frac{1}{\sqrt{1-\frac{\dot{\varphi}^{2}}{T}}}
$$

The EoS parameter for the DBI-essence scalar field model can be written as:

$$
w_{D B I}=\frac{(\eta-1) T(\varphi)-V(\varphi) \eta}{\eta((\eta-1) T(\varphi)+V(\varphi))}
$$

In the present work, we shall assume that $T=a_{1} \dot{\varphi}^{2}$. Using $V(\varphi)=V(\phi)$ in Eq. (3.4) we get reconstructed $w_{D B I}$ that is plotted in Fig. 2. It is evident from the plot that the reconstructed EoS parameter for DBI is -1 during the early phase of the universe and gradually going below -1 at later stage. Hence, it can be said that the EoS is behaving like phantom.

\section{Reconstruction of quintessence dark energy model}

Quintessence is a dynamical, evolving, spatially inhomogeneous component with negative pressure. A common model of quintessence is the energy density associated with a scalar field $Q$ slowly rolling down a potential $V(Q)$. A detailed discussion on quintessence dark energy is available in the review [18]. The energy density and pressure of the quintessence scalar field $\varphi$ are as follow $[19,20,21]$

$$
\begin{aligned}
& \rho_{Q}=\frac{1}{2} \dot{\varphi}^{2}+V(\varphi), \\
& p_{Q}=\frac{1}{2} \dot{\varphi}^{2}-V(\varphi) .
\end{aligned}
$$

The EoS parameter comes out as:

$$
w_{Q}=\frac{\dot{\varphi}^{2}-2 V(\varphi)}{\dot{\varphi}^{2}+2 V(\varphi)}
$$

Using $V(\varphi)=V(\phi)$ in Eq. (4.3) we get reconstructed $w_{\text {quintessence }}$ that is plotted in Fig. 3. Benaviour of the reconstructed EoS parameter appears to be the same as in the case of DBI-essence. 


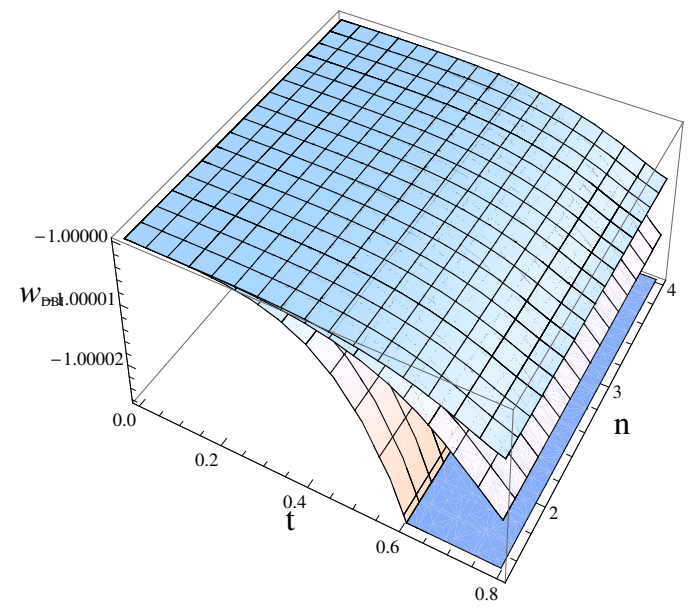

Figure 3: Reconstructed $w_{\text {quintessence }}$ against $t$ for a range of values of $n$.

\section{Concluding remarks}

In the present work we have studied some features of the generalized BD model in which the scalar field is allowed to couple non-minimally with matter sector. Extended holographic Ricci dark energy has been considered in the above framework of BD cosmology. The energy density has been considered in the form $\rho_{\Lambda}=3 \phi\left(\alpha H^{2}+\beta \dot{H}\right)$ to accommodate the BD scalar field in the dark energy density. We have considered $a(t)=a_{0} t^{n}$; and $\phi(t)=\phi_{0} t^{m}$ and reconstructed the coupling function and potential for the extended holographic Ricci dark energy in BD model. We have observed that the potential is increasing with increase in matter-chameleon coupling. Furthermore, we have seen that for any positive value of $m, f(\phi)$ is an increasing function of $t$. This means that for this reconstruction, the coupling function is increasing i.e. the matter-chameleon coupling is getting stronger with passage of cosmic time.

In the next phase we considered correspondence between the reconstructed potential $V(\phi)$ and the potential $V(\varphi)$ for two scalar field DE models namely DBI essence and quintessence. The EoS parameters are reconstructed based on this correspondence. It is observed that in both cases the EoS is behaving like phantom.

Hence, we may conclude that if extended holographic dark energy is considered in the frame of generalized BD model in which the scalar field is allowed to couple non-minimally with matter sector, we can reconstruct the potential of the BD theory. On consideration of correspondence between this potential and potential of scalar field dark energy models, it is possible to attain the phantom phase of the universe in late time. This approach is motivated by [22].

\section{References}

[1] A. G. Riess et al. Astron. J. 116, 1009 (1998).

[2] S. Perlmutter et al. Astrophys. J. 517, 565 (1999).

[3] V. Sahni and A. A. Starobinsky Int. J. Mod. Phys. D 9, 373 (2000) astro-ph/9904398.

[4] V. Sahni and A. A. Starobinsky Int. J. Mod. Phys. D 15, 2105 (2006) astro-ph/0610026. 
[5] H. Motohashi et al. Prog. Theor. Phys. 123, 887 (2010).

[6] P. J. E. Peebles and Ratra, B., Rev. Mod. Phys. 75, 559 (2003) astro-ph/0207347.

[7] E. J. Copeland, M. Sami and S. Tsujikawa Int. J. Mod. Phys. D 15, 1753 (2006).

[8] S. Nojiri, S. D. Odintsov arXiv:0807.0685 (2008).

[9] S. Nojiri, S. D. Odintsov Int. J. Geom. Meth. Mod. Phys. 4, 115 (2007).

[10] C. Brans, R. H. Dicke Phys. Rev. D 124925 (1961).

[11] S. Sen and T. R. Seshadri Int. J. Mod. Phys. D 12, 445 (2003).

[12] H. Alavirad and A. Sheykhi, arXiv:1405.2515 [astro-ph.CO] (2014).

[13] L. N. Granda and A. Oliveros Phys. Lett. B 669275 (2008).

[14] Y. U. Fei and Z. Jing-Fei Commun. Theor. Phys. 59243 (2013).

[15] M. R. Setare and M. Jamil Phys. Lett. B 6901 (2010).

[16] Y. Bisbar Phys. Rev. D 86127503 (2012).

[17] J. Martin, M. Yamaguchi Phys. Rev. D 77123508 (2008).

[18] P. J. Steinhardt Phil. Trans. R. Soc. Lond. A 361, 2497 (2003).

[19] R. R. Caldwell et al. Phys. Rev. Lett. 80, 1582 (1998).

[20] J. P. Wu, D. Z. Ma and Y. Ling Phys. Lett. B 663, 152 (2008).

[21] J. Zhang, X. Zhang and H. Liu Eur. Phys. J. C 54, 303 (2008).

[22] S. Chattopadhyay, A. Pasqua and M. Khurshudyan, Eur. Phys. J. C 74, 3080 (2014). 